\title{
Small Pipes: Preliminary Experience with 3-mm or Smaller Pipeline Flow-Diverting Stents for Aneurysm Repair prior to Regulatory Approval
}

\author{
A.R. Martin, J.P. Cruz, C. O’Kelly, M. Kelly, J. Spears, and T.R. Marotta
}

\begin{abstract}
SUMMARY: Flow diversion has become an established treatment option for challenging intracranial aneurysms. The use of small devices of $\leq 3-\mathrm{mm}$ diameter remains unapproved by major regulatory bodies. A retrospective review of patients treated with Pipeline Embolization Devices of $\leq 3$-mm diameter at 3 Canadian institutions was conducted. Clinical and radiologic follow-up data were collected and reported. Twelve cases were treated with $\geq 1$ Pipeline Embolization Device of $\leq 3$-mm diameter, including 2 with adjunctive coiling, with a median follow-up of 18 months (range, 4-42 months). One patient experienced a posttreatment minor complication (8\%) due to an embolic infarct. No posttreatment hemorrhage or delayed complications such as in-stent stenosis/thrombosis were observed. Radiologic occlusion was seen in $9 / 12$ cases (75\%) and near-occlusion in 2/12 cases (17\%). Intracranial aneurysm treatment with small-diameter flow-diverting stents provided safe and effective aneurysm closure in this small selected sample. These devices should be further studied and considered for regulatory approval.
\end{abstract}

ABBREVIATIONS: PED = Pipeline Embolization Device; PICA = posterior inferior cerebellar artery

$\mathbf{E}$ ndovascular flow diversion by using devices such as the Pipe- line Embolic Device (PED; Covidien, Irvine, California) has gained acceptance as a viable option for endovascular treatment of intracranial aneurysms not amenable to more conventional therapies. This technique has been established mainly in the treatment of proximal unruptured aneurysms in relatively large parent vessels, with a paucity of data describing the use of small devices. ${ }^{1}$ Health Canada has only approved PED use in parent vessels of $\geq 3.25 \mathrm{~mm}$ in diameter, ${ }^{2}$ perhaps due to concern that smaller devices might be prone to complications such as access difficulties, kinking, or in-stent thrombosis. The US FDA did not specify a size constraint in its approval letter but specified that the PED was only approved for ICA aneurysms from the petrous segment to the superior hypophyseal segment. ${ }^{3}$ This limited approval also effectively restricts PED use to larger devices because it is extremely rare for the ICA to measure $<3 \mathrm{~mm}$. Furthermore, distalvessel aneurysms frequently have wide-neck or fusiform morphology, making them difficult to treat with conventional

Received April 8, 2014; accepted after revision August 23.

From the Division of Neurosurgery (A.R.M., J.S.), Department of Surgery, and Department of Radiology (J.P.C., T.R.M.), University of Toronto, Toronto, Ontario, Canada; Division of Neurosurgery (C.O.), Department of Surgery, University of Alberta, Edmonton, Alberta, Canada; and Division of Neurosurgery (M.K.), Department of Surgery, College of Medicine, University of Saskatchewan, Saskatoon, Saskatchewan, Canada.

Please address correspondence to Allan R. Martin, MD, 4th Floor, West Wing, 399 Bathurst St, Toronto, Ontario, Canada, M5T2S8; e-mail: allan.martin@utoronto.ca

三 Indicates article with supplemental on-line table.

http://dx.doi.org/10.3174/ajnr.A4170 techniques such as coiling and potentially good candidates for flow diversion. Therefore, it is imperative that the safety profile of small flow-diversion devices be well-studied so that challenging aneurysms in small-diameter parent vessels can be properly evaluated for potential use of this emerging treatment option.

\section{MATERIALS AND METHODS}

We conducted a retrospective review at 3 institutions of all endovascular cases using PEDs between June 2008 and July 2013. Patients that were treated with one or more small stents $(\leq 3.00 \mathrm{~mm}$ in diameter) were included in our analysis. Data including demographics; aneurysm type, size, and location; procedural details; clinical presentation; subsequent imaging; and clinical outcome, including the most recent follow-ups, were collected. Our results are expressed in medians and interquartile ranges, given the small sample size (non-normal distribution).

The decision to treat was made for each case by a multidisciplinary team, including vascular neurosurgeons and interventional neuroradiologists, and Health Canada approval was individually obtained under an appeal for compassionate use. Informed consent was obtained from patients or substitute decision-makers. Pretreatment antiplatelet therapy included both acetylsalicylic acid $(325 \mathrm{mg}$ ) and a total dose of $600 \mathrm{mg}$ of clopidogrel before the procedure (initiated 5 days prior for unruptured aneurysms or within 24 hours for ruptured aneurysms). Testing of in vitro platelet function for clopidogrel response was not performed (not approved by Health Canada). In cases presenting with SAH, extraventricular drains were placed before initiation of 


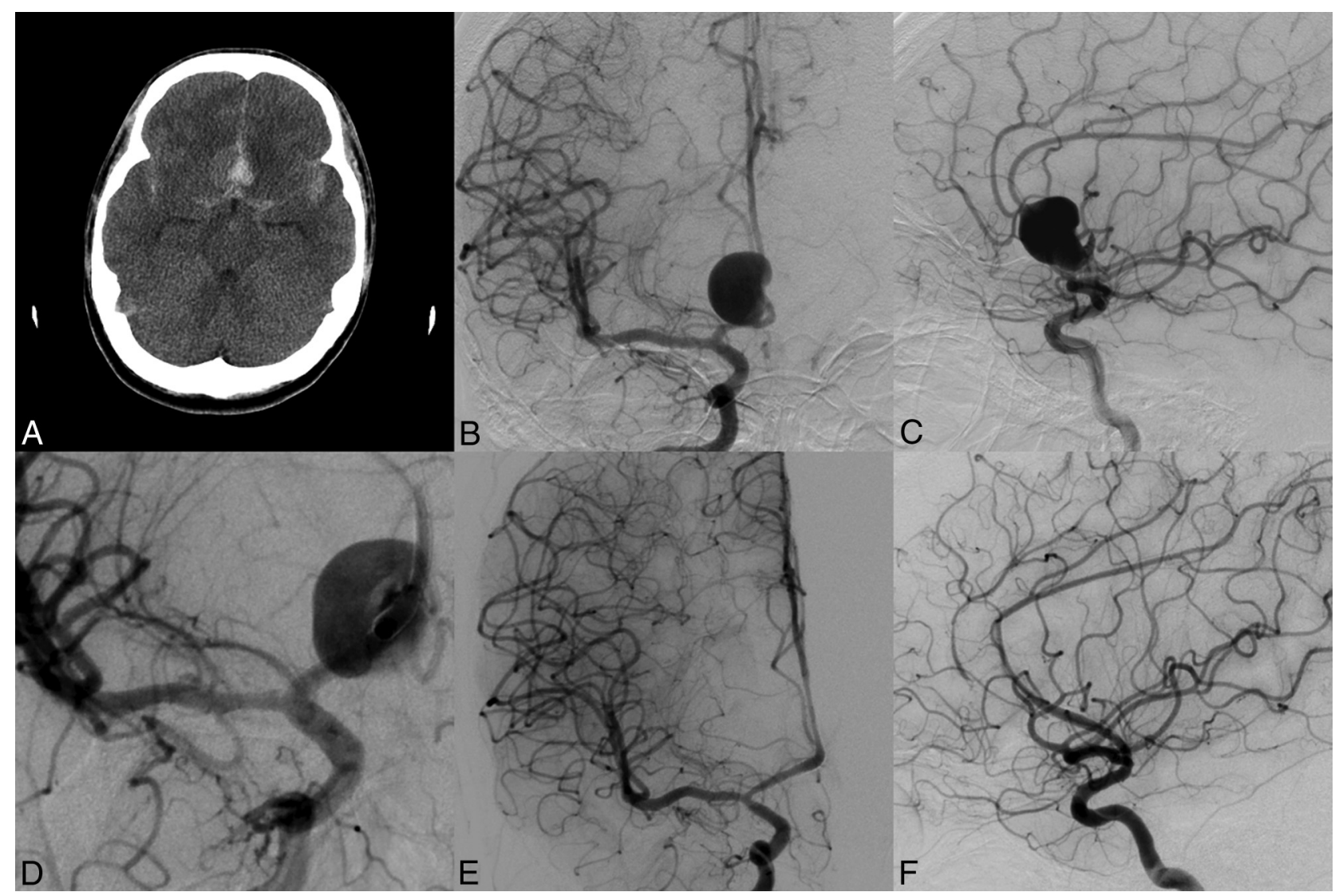

FIG 1. Representative images for case 6. A, Noncontrast CT demonstrates an acute SAH centered in the interhemispheric fissure. $B$ and $C$, Anteroposterior and lateral projections, right ICA injections, pretreatment, show a right A2 fusiform aneurysm. $D$, Oblique projection, right ICA injection, immediately postdeployment, shows the flow effect and position of the stent. $E$ and $F$, Anteroposterior and lateral projections, right ICA injections, at 2 months posttreatment show complete aneurysm occlusion.

dual antiplatelet therapy if indicated. Intraprocedural heparin was administered to achieve a targeted activated clotting time of 250-300 seconds.

All procedures were performed with the patient under general anesthesia in a biplane angiography suite. Standard transfemoral access was obtained, and a triaxial approach was used for all anterior circulation aneurysms and for those posterior circulation aneurysms in which the dominant vertebral artery was of sufficient size to allow these devices. A 0.027-inch microcatheter (Marksman; Covidien) was used to gain a distal position across the neck of the aneurysm. In cases in which adjuvant coiling was planned, a 0.014 - or 0.018 -inch microcatheter was placed into the aneurysm lumen before stent deployment, by using a 5F guide catheter via contralateral femoral access. PED sizes were selected on the basis of the proximal and distal diameters of the parent vessel. One or more PEDs were then deployed to reconstruct the parent artery, depending on the degree of inflow reduction. In coiling cases, loose-packed coils would be placed until the primary operator was satisfied with the result.

All patients were monitored postprocedure in a dedicated neurosurgical intensive care unit. Patients typically underwent postprocedural MRA within 48 hours. After discharge, clinical and imaging follow-up varied on the basis of the treating physician's discretion and the patient's wishes. Radiologic follow-up consisted of DSA or noninvasive imaging (MRA or CTA) and was usually performed at 4-6 months. Dual antiplatelet therapy was strictly continued for a minimum of 6 months. After this, discontinuation of clopidogrel was based on imaging findings and the discretion of the treating physician, and the patient was maintained on aspirin only.

\section{RESULTS}

The results are summarized in the On-line Table, with representative images displayed in Figs 1 and 2. The median diameter was $18 \mathrm{~mm}$ (range, 2-38 mm). Among the aneurysms treated, 6/12 (50\%) were located in the anterior circulation, and 6/12, (50\%) in posterior circulation (Table). Three patients were treated in the acute phase of SAH; 1 patient, in the subacute phase; and 1, with remote SAH. Five patients had previous treatment with coiling or a standalone stent and experienced subsequent recanalization before their treatment with flow diversion. Two patients (17\%) were treated with both coiling and flow diversion.

One patient $(8 \%)$ experienced technical complications with access and required prestenting angioplasty of the parent vessel so that the device could cross the aneurysm. One patient (8\%) experienced a clinically significant procedure-related complication, with a distal posterior inferior cerebellar artery (PICA) stroke, which was detected in the immediate postprocedure period with mild dysmetria and limb ataxia, which improved to a minimal deficit during several days. This stroke was seen on postprocedure MR imaging, and the mechanism appeared to be embolic on the basis of its distal territory, most likely occurring during catheter 


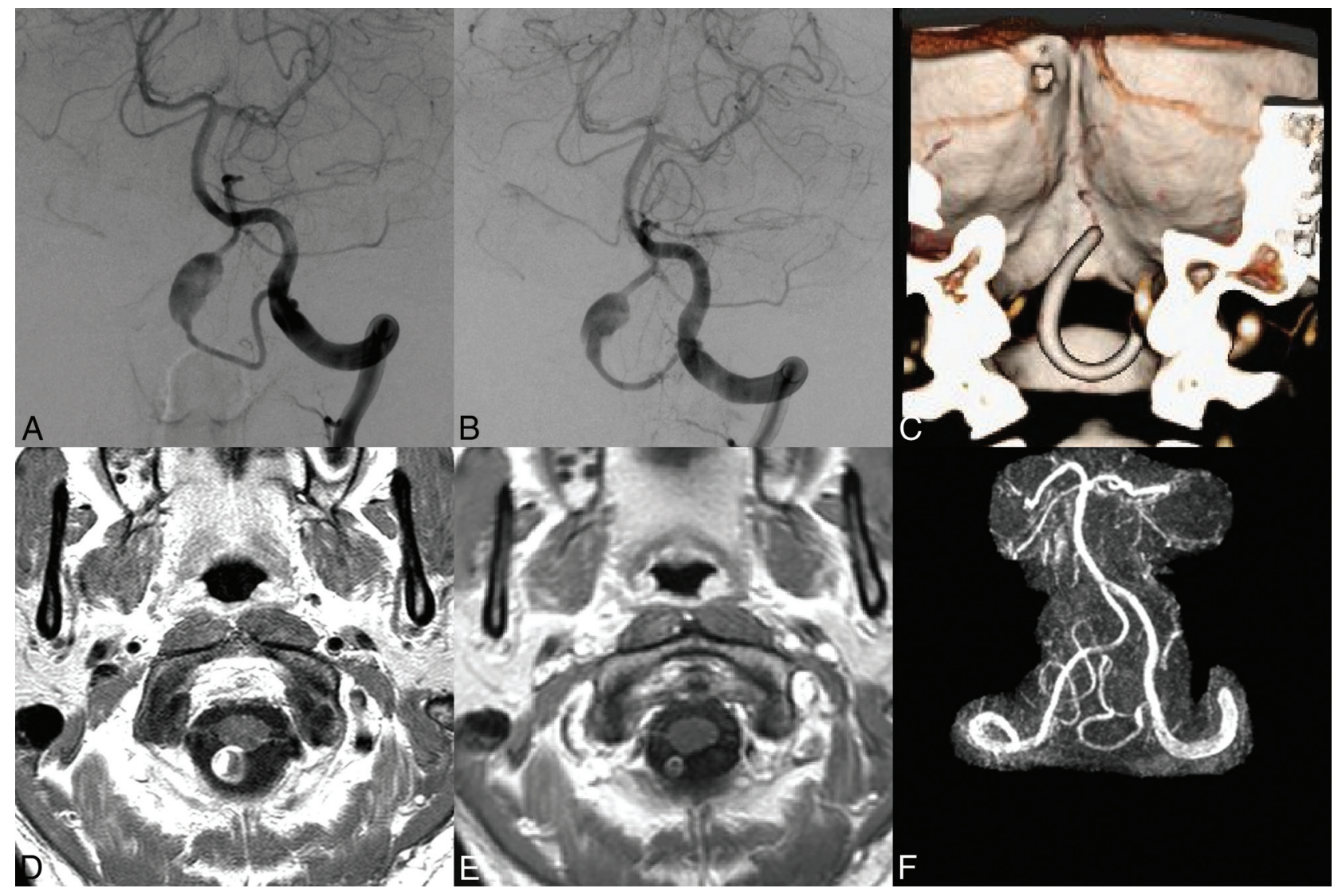

FIG 2. Representative images for case 7. $A$ and $B$, Anteroposterior projections, left vertebral artery injections, pretreatment and immediately post-PED deployment, respectively, show persistent filling with a minor flow effect. C, 3D reconstruction of CTA at 4 months postprocedure shows placement of the stent entirely in the PICA. D and E, Axial T1 MR imaging with gadolinium, postoperative day 1 and at 1-year follow-up, respectively, shows delayed aneurysm occlusion. $F, 3 D$ reconstruction of the MRA at 1-year follow-up shows patency of the parent vessel.

\section{Summary of aneurysm characteristics by size/location}

\begin{tabular}{ccccc}
\hline Location & $<\mathbf{~} \mathbf{~ m m}$ & $\mathbf{7 - 1 2} \mathbf{~ m m}$ & $\mathbf{1 3 - 2 4} \mathbf{~ m m}$ & $>\mathbf{2 4} \mathbf{~ m m}$ \\
\hline Anterior (ICA/MCA/ACA) & 2 & 1 & 2 & 1 \\
$\begin{array}{l}\text { Posterior (vertebrobasilar, } \\
\text { PcomA, PCA) }\end{array}$ & 1 & 2 & 2 & 1 \\
\hline
\end{tabular}

Note:-PcomA indicates posterior communicating artery; PCA, posterior cerebral artery; ACA, anterior cerebral artery.

navigation, because the aneurysm was located at the superior cerebellar artery takeoff. One additional patient (8\%) had clinically asymptomatic infarcts seen on postprocedural MR imaging (day 1 ), not requiring any specific treatment or rehabilitation. This patient was further assessed by early DSA, and it was found that the frontopolar artery, which was covered by the PED, was now occluded, whereas it was patent in the immediate postdeployment angiogram. No patients had a periprocedural decrease in the Glasgow Outcome Score. No delayed complications were seen at a median follow-up of 1.5 years (range, 6 months to 3.5 years), including no cases of in-stent stenosis/thrombosis or rerupture.

The results at latest follow-up included a complete occlusion rate of $9 / 12(75 \%)$, a near-occlusion rate of $2 / 12(17 \%)$, and a residual filling rate of $1 / 12(8 \%)$. The timing of follow-up imaging and the choice of technique were variable, with 5/12 (42\%) undergoing only noninvasive imaging postprocedure. Among the cases that showed complete occlusion, the median time for first documentation of occlusion was 6 months (range, 1 day to 8 months). The 2 cases of near-occlusion were both first documented at 6 months. The case that had residual filling was documented on MRA at 1 day, and this was persistently seen on several follow-up studies including DSA at 1.5 years and MRA at 2.5 years.

\section{DISCUSSION}

The use of $\leq 3-\mathrm{mm}$ diameter flow-diversion devices is not currently approved in North America, and there is a paucity of published literature that describes their use. Pistocchi et al ${ }^{1}$ reported a series of 26 patients, most of whom were treated with flow-diversion devices of $\leq 3 \mathrm{~mm}$, with good outcomes including an occlusion rate of $83 \%$ and a neurologic complication rate of $4 \%$ with no hemorrhage. Yavuz et $\mathrm{al}^{4}$ recently reported 25 cases of MCA aneurysms located at the bifurcation or more distally, with an occlusion rate of $84 \%$ and only 1 case of long-term neurologic impairment, which was mild (mRS 1). Presumably, many of the cases in this series involved small-diameter parent vessels and PEDs, but these specific data were not reported. Most larger flow-diversion series either did not report any cases of devices of $\leq 3 \mathrm{~mm}$, or they did not include documentation of the parent vessel or device size. ${ }^{4,6-8}$ Our current series of 12 patients demonstrated that the use of these small devices is technically feasible and safe, with excellent clinical and radiologic outcomes at a median latest follow-up of 1.5 years. The rates of occlusion $(75 \%)$ and near- or complete occlusion (92\%) are similar to overall data published for flow-diversion treatment (76\% complete occlusion at 1 year), ${ }^{9}$ and our current series comprises $50 \%$ posterior circulation aneu- 
rysms, which are known to carry higher rates of complications. The early morbidity rate of $8 \%$ is similar to the established $5 \%-7 \%$ seen in overall data, ${ }^{9,10}$ and no aneurysm ruptures or delayed complications were observed among these data, whereas published data suggest a late morbidity of $3 \%$ and mortality of $1 \% .^{10}$ Therefore, the overall safety profile of this small series appears grossly in line with the results of Pistocchi et $\mathrm{al}^{1}$ and the overall published flow-diversion data. ${ }^{4-10}$

The timing of follow-up imaging and the choice of imaging technique varied greatly among the cases in this series, on the basis of the discretion of the treating clinician. It was thought that performing follow-up DSA was unnecessary in cases with good clinical status and a satisfactory noninvasive radiologic result. In cases without any coils present, it was thought that CTA was the superior noninvasive technique in assessing in-stent stenosis, but MRA was generally acceptable for assessing patency. For cases with any concerning features such as suspected stenosis, DSA remains the optimal technique for detailed assessment.

The use of flow diversion in small and/or distal vessels has unique technical challenges that may increase the risk of ischemic or hemorrhagic complications during both access and deployment. Access in small and/or distal vessels is likely to be more difficult in comparison with that in larger and/or proximal flow diversion because the device is delivered in a large and relatively stiff microcatheter and tortuosity or sharp corners can pose serious roadblocks. Establishing a distal position with a microwire sometimes requires using a larger microwire ( 0.018 inch) or a buddy wire technique (0.008/0.010 and 0.014 inch). The distal landing zone may be of particularly small caliber, increasing the risk of dissection or perforation by the wire or the microcatheter. Smaller vessels also pose a risk of clot formation or distal spasm because the microcatheter may slow or occlude flow. These risks are also of concern during deployment when the pusher wire may appear in small distal branches. However, our early experience did not demonstrate an increased risk of vessel dissection, perforation, or spasm.

Another technical challenge is deploying the device with a smaller margin of error on the proximal and distal landing zones, because the surrounding vessels are likely to have nearby branches. Proper sizing becomes a critical factor in smaller vessels to maximize coverage across the aneurysm but avoid too much coverage over branches/perforators because fully expanded devices provide higher surface area coverage in small vessels. In addition, we found that it was sometimes more difficult to unscrew the release coil while maintaining the intended delivery position in these smaller devices than in their larger counterparts, because the proximal vascular loops decrease the torque of the delivery system. However, all of these issues were found to be manageable, and difficulties diminished as greater experience deploying small PEDs was acquired.

An important topic in flow diversion is the covering, or jailing, of sidewall or branch arteries by the flow-diverting stent. This topic has been widely discussed in the flow-diversion literature, with the consensus suggesting that branch occlusions are not uncommon but are often clinically silent. ${ }^{5,11,12}$ It remains unclear whether small-diameter flow diverters have a greater propensity to cause occlusion or symptomatic flow reduction to covered arterial branches, but the series by Yavuz et $\mathrm{al}^{4}$ showed relatively high rates of branch occlusion $(3 / 21,14 \%)$ and a reduced caliber of the covered branch $(6 / 21,29 \%)$ among 21 MCA bifurcation aneurysms treated. ${ }^{12}$ Our series demonstrated 1 case $(8 \%)$ of a jailed branch artery occlusion (frontopolar artery) with an associated area of diffusion restriction on MR imaging (postprocedure day 1) suggestive of infarct, but this was clinically silent. There was no radiologic evidence in our series of other infarcts or significant branch occlusions on MR imaging or DSA that were attributable to jailed branches, but in the retrospective review of our cases, it was difficult to accurately determine the patency of smaller jailed branches and perforators. Future study of flow diversion in small vessels would likely benefit from a careful analysis of all jailed vessels, such as a comparison among predeployment, immediate postdeployment, and delayed follow-up by using 3D DSA or 2D images with precisely matched projections.

In-stent stenosis and thrombosis have been cited as important concerns with flow-diversion devices, which occur in $5 \%-10 \%$ of cases, ${ }^{9}$ and these may be even more of a concern with small devices because there is less room available for stenosis to occur before complete thrombosis occurs. ${ }^{13}$ Pistocchi et al $^{1}$ reported 1 case of in-stent thrombosis (4\%) in their series, which was treated with angioplasty in a subsequent procedure that restored flow, but the patient still had a clinical infarct. Delayed ischemia due to intimal hyperplasia is also a possibility, and if this occurs so that a vessel diameter is reduced by $1-2 \mathrm{~mm}$, it could theoretically lead to a critical flow effect in stents of $<3 \mathrm{~mm}$. Furthermore, in-stent stenosis may be more difficult to detect in small-caliber stents due to a lack of imaging resolution and the presence of metal artifacts. In our series, we did not observe any cases of significant in-stent stenosis and there were no cases of thrombosis, but further data are needed to know whether these small devices pose a greater risk of this complication.

The use of intravascular metal stents requires dual antiplatelet therapy, which is commonly recommended for at least 6 months before reduction to a single antiplatelet agent, though variations in practice exist. ${ }^{8}$ The preprocedural regimen and postprocedural duration remain controversial, especially in the setting of SAH. Our series included 3 cases that were treated in the acute phase of $\mathrm{SAH}$ with none of these experiencing early rerupture, similar to previous results. ${ }^{14,15}$ The optimal timing likely depends on the degree of epithelialization of the metal device, which, in turn, depends on various factors such as flow characteristics, aneurysm occlusion, length, and the presence of endoleaks. ${ }^{11,16}$ All of these variables may be even more important in small-sized vessels. One possibility is that higher resolution imaging such as vessel wall MRA will eventually be able to provide evidence of epithelialization, but until then, controversy will likely continue.

\section{CONCLUSIONS}

Flow diversion with small-diameter devices $(\leq 3 \mathrm{~mm})$ remains unapproved by Health Canada, and the US FDA effectively imposes a similar constraint. Our current results document the safe and effective use of small-diameter flow diverters in a small selected sample, without evidence of higher-than-published complication rates such as in-stent stenosis/thrombosis, dissection, or rerupture. As such, this treatment option should be further studied and potentially considered for broader regulatory approval. 
Disclosures: Cian O'Kelly_RELATED: Consulting Fee or Honorarium: Covidien, Comments: proctor for Pipeline cases; UNRELATED: Grants/Grants Pending: National Institutes of Health trial Minimally Invasive Surgery plus rt-PA for ICH Evacuation, ${ }^{*}$ Comments: due to start October 2014; we receive an amount to our institution per patient enrolled. Michael Kelly—RELATED: Support for Travel to Meetings for the Study or Other Purposes: Covidien, ${ }^{\star}$ Comments: 2014 Flow Diversion Meeting, Toronto, Ontario, Canada (April 10, 2014); OTHER RELATIONSHIPS: I am a shareholder of Blockade Medical, Inc. Thomas R. Marotta-RELATED: Consulting Fee or Honorarium: proctor for Pipeline (Canada); Fees for Participation in Review Activities such as Data Monitoring Boards, Statistical Analysis, Endpoint Committees, and the Like: Flow Redirection Endoluminal Device study, China Endovascular Course, Low-profile Visualized Intraluminal Support device study data and safety monitoring board; UNRELATED: Consultancy: As above, Pipeline proctor; Patents (planned, pending or issued): My invention, eclips, endovascular clip systems, flow diverter for bifurcation aneurysms. *Money paid to the institution.

\section{REFERENCES}

1. Pistocchi S, Blanc R, Bartolini B, et al. Flow diverters at and beyond the level of the circle of Willis for the treatment of intracranial aneurysms. Stroke 2012;43:1032-38

2. Health Canada. Summary of basis of decision: Pipeline embolization device. http://www.hc-sc.gc.ca/dhp-mps/prodpharma/sbd-smd/ md-im/sbd_smd_2010_pipeline_embolization_148227-eng.php. Accessed March 24, 2014

3. US Food and Drug Administration. Pipeline embolization device approval letter. http://www.accessdata.fda.gov/cdrh_docs/pdf10/ p100018a.pdf. Accessed March 24, 2014

4. Yavuz K, Geyik S, Saatci I, et al. Endovascular treatment of middle cerebral artery aneurysms with flow modification with the use of the Pipeline embolization device. AJNR Am J Neuroradiol 2014;35:529-35

5. Fiorella D, Woo HH, Albuquerque FC, et al. Definitive reconstruction of circumferential, fusiform intracranial aneurysms with the Pipeline embolization device. Neurosurgery 2008:62:1115-20; discussion 1120-21

6. Kulcsár Z, Wetzel SG, Augsburger L, et al. Effect of flow diversion treatment on very small ruptured aneurysms. Neurosurgery 2010; 67:789-93

7. Deutschmann HA, Wehrschuetz M, Augustin M, et al. Long-term follow-up after treatment of intracranial aneurysms with the Pipeline embolization device: results from a single center. AJNR Am J Neuroradiol 2012;33:481-86

8. McAuliffe W, Wenderoth JD. Immediate and midterm results following treatment of recently ruptured intracranial aneurysms with the Pipeline embolization device. AJNR Am J Neuroradiol 2012;33:487-93

9. Brinjikji W, Murad MH, Lanzino G, et al. Endovascular treatment of intracranial aneurysms with flow diverters: a meta-analysis. Stroke 2013;44:442-47

10. Arrese I, Sarabia R, Pintado R, et al. Flow-diverter devices for intracranial aneurysms: systematic review and meta-analysis. Neurosurgery 2013;73:193-99; discussion 199-200

11. Kallmes DF, Ding YH, Dai D, et al. A new endoluminal, flow-disrupting device for treatment of saccular aneurysms. Stroke 2007;38:2346-52

12. Saatci I, Yavuz K, Ozer C, et al. Treatment of intracranial aneurysms using the Pipeline flow-diverter embolization device: a single-center experience with long-term follow-up results. AJNR Am J Neuroradiol 2012;33:1436-46

13. Liou TM, Li YC. Effects of stent porosity on hemodynamics in a sidewall aneurysm model. J Biomech 2008;41:1174-83

14. Martin AR, Cruz JP, Matouk CC, et al. The Pipeline flow-diverting stent for exclusion of ruptured intracranial aneurysms with difficult morphologies. Neurosurgery 2012;70(1 suppl operative):21-28; discussion 28

15. Cruz JP, O'Kelly C, Kelly M, et al. Pipeline embolization device in aneurysmal subarachnoid hemorrhage. AJNR Am J Neuroradiol 2013;34:271-76

16. Dai D, Ding YH, Kadirvel R, et al. Patency of branches after coverage with multiple telescoping flow-diverter devices: an in vivo study in rabbits. AJNR Am J Neuroradiol 2012;33:171-74 